Hutzelmeyer-Nickels, Anna; Noterdaeme, Michele

\title{
Anwendbarkeit der Child Behavior Checklist 11/2-5 als orientierendes Untersuchungsinstrument bei Vorschulkindern mit Entwicklungsproblemen
}

Praxis der Kinderpsychologie und Kinderpsychiatrie 56 (2007) 7, S. 573-588

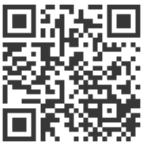

Quellenangabe/ Reference:

Hutzelmeyer-Nickels, Anna; Noterdaeme, Michele: Anwendbarkeit der Child Behavior Checklist 1 1/2-5 als orientierendes Untersuchungsinstrument bei Vorschulkindern mit Entwicklungsproblemen - In: Praxis der Kinderpsychologie und Kinderpsychiatrie 56 (2007) 7, S. 573-588 - URN:

urn:nbn:de:0111-opus-30564 - DOI: 10.25656/01:3056

https://nbn-resolving.org/urn:nbn:de:0111-opus-30564

https://doi.org/10.25656/01:3056

in Kooperation mit / in cooperation with:

\section{Vandenhoeck \& Ruprecht v\&R}

http://www.v-r.de

\section{Nutzungsbedingungen}

Gewährt wird ein nicht exklusives, nicht übertragbares, persönliches und beschränktes Recht auf Nutzung dieses Dokuments. Dieses Dokument is ausschließlich für den persönlichen, nicht-kommerziellen Gebrauch bestimmt. Die Nutzung stellt keine Übertragung des Eigentumsrechts an diesem Dokument dar und gilt vorbehaltlich der folgenden Einschränkungen: Auf sämtlichen Kopien dieses Dokuments müssen alle Urheberrechtshinweise und sonstigen Hinweise auf gesetzlichen Schutz beibehalten werden. Sie dürfen dieses Dokument nicht in irgendeiner Weise abändern, noch dürfen Sie dieses Dokument für öffentliche oder kommerzielle Zwecke vervielfältigen, öffentlich ausstellen, aufführen, vertreiben oder anderweitig nutzen.

Mit der Verwendung dieses Dokuments erkennen Sie die Nutzungsbedingungen an.

\section{Terms of use}

We grant a non-exclusive, non-transferable, individual and limited right to using this document.

This document is solely intended for your personal, non-commercial use. Use of this document does not include any transfer of property rights and it is conditional to the following limitations: All of the copies of this documents must retain all copyright information and other information regarding legal protection. You are not allowed to alter this document in any way, to copy it for public or commercial purposes, to exhibit the document in public, to perform, distribute or otherwise use the document in public.

By using this particular document, you accept the above-stated conditions of use.

\section{Kontakt / Contact:}

peDOCs

DIPF | Leibniz-Institut für Bildungsforschung und Bildungsinformation Informationszentrum (IZ) Bildung

E-Mail: pedocs@dipf.de

Internet: www.pedocs.de

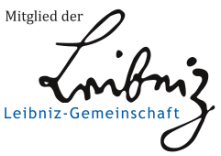




\title{
AUS KLINIK UND PRAXIS
}

\section{Anwendbarkeit der Child Behavior Checklist 11/2-5 als orientierendes Untersuchungsinstrument bei Vorschulkindern mit Entwicklungsproblemen}

\author{
Anna Hutzelmeyer-Nickels und Michele Noterdaeme
}

\begin{abstract}
Summary
Usefulness of the Child Behavior Checklist in the assessment of preschool children with developmental problems

The diagnostic evaluation of developmental problems and behavior problems in early childhood is difficult and time consuming. This study analyses the usefulness of the Child Behavior Checklist (CBCL) as a screening instrument in a special clinic for children with pervasive and specific developmental disorders. 106 children under the age of 6 years were enrolled in the study. All children were individually assessed. Psychiatric diagnoses were based on parent interview, observation of the child, speech and language assessment as well as cognitive assessment and extensive neurological examination. In addition, behavior and emotional problems were recorded with the CBCL. The results show that less than half of the children with a psychiatric diagnosis had a total score (CBCL) above the cut-off value. However, all children with a total score in the clinical range had a psychiatric diagnosis. The syndrome scales and diagnostic scales are not sensitive. The diagnostic scale "pervasive developmental disorder" is not specific enough to be used as a reliable instrument. In order to improve the usefulness of the checklist, a adjustment of the cut-off values should be considered.
\end{abstract}

Prax. Kinderpsychol. Kinderpsychiat. 56/2007, 573-588

\section{Keywords}

developmental disorder - preschool - CBCL - behavioral problems

\section{Zusammenfassung}

Auffälligkeiten in der Entwicklung und im Verhalten in der frühen Kindheit sind schwierig zu diagnostizieren und erfordern eine zeitaufwändige kinderpsychiatrische Untersuchung. In der hier vorgelegten Arbeit wird untersucht, inwiefern sich der Fragebogen „Child Behavior Checklist für Klein- und Vorschulkinder" (CBCL 1/1/2-5) als orientierendes Untersuchungsinstrument bei der diagnostischen Abklärung von Vorschulkindern in einer kinderpsychiatri- 
schen Ambulanz mit dem Schwerpunkt Entwicklungsstörungen eignet. Eine Stichprobe von 106 Kindern unter 6 Jahren wurde mit 2 Methoden untersucht. Erstens erfolgte eine umfassende klinische kinderpsychiatrische Diagnostik nach dem multiaxialen Schema, zweitens füllten die Eltern jeweils den Fragebogen CBCL 11/2-5 aus. Die Ergebnisse zeigten, dass sich der Einsatz der CBCL 1 1/2-5 als nur bedingt sinnvoll erweist. Weniger als die Hälfte der Kinder mit einer klinisch psychiatrischen Diagnose hatte einen auffälligen Gesamtwert in der CBCL 1⁄1/2-5. Andererseits erwies der Gesamtwert eine gute Spezifität, ein auffälliger Gesamtwert spricht also mit hoher Wahrscheinlichkeit für eine psychiatrische Diagnose. Die Problemskalen und Diagnoseskalen (außer der Skala „Tiefgreifende Entwicklungsstörung“) zeigten eine geringe Sensitivität, erfassten aber die Kernsymptomatik der jeweiligen Diagnosegruppe.

\section{Schlagwörter}

Entwicklungsstörung - Vorschulalter - CBCL - Verhaltensstörungen

\section{$1 \quad$ Einleitung}

Bereits im Vorschulalter werden viele Kinder aufgrund von Verhaltensproblemen oder Auffälligkeiten in ihrer Entwicklung ambulant in der Kinder- und Jugendpsychiatrie vorgestellt. Die Häufigkeit von Verhaltensproblemen im Vorschulalter wird von Pouštka (1993) gemäß verschiedener Studien mit 10\% angegeben. Zudem sind bereits im Kindergartenalter komorbide Störungen häufig, die Braunschweiger Kindergartenstudie ermittelte bidirektionale Komorbiditätsraten zwischen knapp 11 und 25\% (Kuschel et al., 2004). Zu den häufigsten Verhaltensstörungen im Vorschulalter zählen hyperkinetische- und Sozialverhaltensstörungen (Wolff Metternich et al., 2002). Die Prävalenz einer hyperkinetischen Störung wird in klinischen Studien mit 2 bis 9,5\% (Steinhausen, 2002), einer Sozialverhaltensstörung mit oppositionellem Trotzverhalten mit 7 bis $25 \%$ (Wolff Metternich u. Döpfner, 2000) angegeben. Neben expansiven Verhaltensweisen berichten Eltern von Vorschulkindern häufig über emotionale Probleme, wie ausgeprägte Ängste oder depressive Symptome (Resch, 1996). In der Befragung von Eltern und Erziehern von Vorschulkindern durch Wolff Metternich und Döpfner (2000) wurden bei 2,5\% der Kinder ausgeprägte emotionale Auffälligkeiten geschildert. Andere Verhaltensauffälligkeiten im Vorschulalter sind Probleme im sozialen Kontakt, Probleme des Essverhaltens, Schwierigkeiten der Ausscheidungsfunktionen, Schlafprobleme und Verhaltensweisen der Selbsttröstung (Goodman et al., 2000). Ein seltenes Störungsbild der sozialen Interaktion und Kommunikation, das sich aber bereits vor dem 3. Lebensjahr manifestiert (Remschmidt u. Schmidt, 2001), ist der frühkindliche Autismus. Autistische Störungen zählen zu den tiefgreifenden Entwicklungsstörungen. Der frühkindliche Autismus wird definiert durch eine gestörte Funktionsfähigkeit der sozialen Interaktion, der Kommunikation und ein eingeschränktes, repetitives Verhalten. Die Prävalenz des frühkindlichen Autismus wird auf 1 bis 2 pro 1000 Personen geschätzt (Noterdaeme et al., 2002).

(c) Vandenhoeck \& Ruprecht GmbH \& Co. KG, Göttingen 2007 
Nicht selten verbergen sich hinter Verhaltensproblemen bei Vorschulkindern umschriebene Entwicklungsstörungen. Umschriebene Entwicklungsstörungen kennzeichnen isolierte Defizite einzelner Funktionsbereiche, die aufgrund der allgemeinen Begabung, Förderung sowie körperlichen und seelischen Gesundheit des Betroffenen nicht erklärt werden können (Esser u. Wyschkon, 2002). Dazu zählen umschriebene Entwicklungsstörungen des Sprechens und der Sprache sowie der motorischen Funktionen. Gemäß DSM-IV wird die Rate expressiver Sprachstörungen auf 3 bis 5\% und rezeptiver Sprachstörungen auf 3\% geschätzt (APA, 2000). Störungen im sprachlichen Bereich sind häufig mit kinderpsychiatrischen Problemen assoziiert (Suchodoletz, 2003). Bei mehr als 50\% der betroffenen Kinder treten weitere Teilleistungsstörungen auf. Mehr als die Hälfte der Kinder mit einer Sprachverständnisstörung zeigen eine komorbide psychiatrische Störung (Noterdaeme et al., 1999). Bei einem Teil der Kinder werden die psychiatrischen Probleme schon im Vorschulalter deutlich. $\mathrm{Zu}$ den häufigsten Problemen zählt die hyperkinetische Störung, gefolgt von emotionalen Störungen mit Rückzug, Ängstlichkeit, Tagträumen und einer Störung des Sozialverhaltens. Bei jüngeren Kindern werden des Weiteren multiple Tics, Enuresis und Enkopresis angeführt (Amorosa u. Noterdaeme, 1999).

Prospektive Studien zeigen, dass Verhaltensprobleme im Kleinkind und Vorschulalter häufig persistierenden Charakter haben (McDonell u. Glod, 2003). Campbell (1995) zufolge weisen 50\% der Kinder, die mit 3 Jahren Verhaltensschwierigkeiten haben, diese auch noch im Schulalter auf. Expansive Störungen wie Hyperaktivität oder Störungen des Sozialverhaltens zeigen eine höhere Stabilität als emotionale Beeinträchtigungen. Besonders frühe expansive Störungen bergen ein hohes Risiko in sich, einem ungünstigen Entwicklungsverlauf Vorschub zu leisten (Resch, 1996). Campbell (1995) berichtete, dass 68\% der Kinder, die mit 3 Jahren expansives Problemverhalten zeigten, auch noch mit 6 und 9 Jahren auffällig blieben.

Um einer ungünstigen Entwicklung entgegen zu wirken, ist eine frühe Diagnosestellung und Einleitung therapeutischer Maßnahmen besonders wichtig. Eine multimodale kinderpsychiatrische Diagnostik im Vorschulalter stützt sich neben Anamnese und Verhaltensbeobachtung auch auf verschiedene Testverfahren zur Beurteilung des aktuellen Entwicklungs- und Leistungsstandes. In der Anamneseerhebung finden neben der Befragung von Eltern und anderen Bezugspersonen standardisierte Fragebogenverfahren zur Verhaltens- und Psychodiagnostik als Baustein eines multimodalen Diagnostikprozess breite Anwendung. $\mathrm{Zu}$ den wichtigsten deutschsprachigen Basisverfahren bei Kindern bis zum Schulalter zählt die Child Behavior Checklist für Klein- und Vorschulkinder (CBCL 1²-5). Fragebogenbasisverfahren decken ein weites Spektrum psychischer Auffälligkeiten und psychosozialer Kompetenzen ab. In der anonymeren Fragebogensituation werden Problembereiche evtl. bereitwilliger eingeräumt als in der persönlichen Exploration (Döpfner et al., 2000). Verfahren mit Fragebogen sind in der Regel ohne größeren Zeitaufwand durchführbar, z. B. während der Wartezeit (Herpertz-Dahlmann et al., 2003). 
Ziel der hier vorgelegten Arbeit ist es, weitere Erkenntnisse über Art, Häufigkeit und Komorbidität bei Vorschulkindern mit Verhaltens- und Entwicklungsstörungen zu gewinnen. Insbesondere sollte festgestellt werden inwiefern der Fragebogen CBCL 1 1 $12-5$ die klinische Diagnostik vereinfachen oder sinnvoll ergänzen kann. Als Fragebogenbasisverfahren sollte sie den Verdacht auf einzelne Störungsbilder lenken und Anlass zu gezielter Exploration geben.

\section{Methodik}

Eine Stichprobe von 106 Kindern unter 6 Jahren wurde mit 2 Methoden untersucht. Erstens wurde eine klinische Diagnostik durch ein multiprofessionelles Team, bestehend aus Kinderpsychiatern, Psychologen, Sprach- und Mototherapeuten durchgeführt. Die Kinder wurden anhand der klinisch-psychiatrischen Diagnose in vier Diagnosegruppen unterteilt. Zweitens füllten die Eltern für alle Kinder den Fragebogen CBCL 1 1/2-5 aus. Die Ergebnisse beider Methoden wurden miteinander verglichen. Dazu wurde mit statistischen Analyseverfahren überprüft, inwiefern die CBCL zwischen den vier klinisch ermittelten Diagnosegruppen unterscheidet.

\subsection{Stichprobe}

In der Spezialambulanz der Heckscher Klinik werden Kinder mit schweren umschriebenen Entwicklungsrückständen (Sprachstörungen, Lese-Rechtschreibstörungen, motorischen Störungen), tiefgreifenden Entwicklungsstörungen sowie Verhaltensauffälligkeiten diagnostisch abgeklärt und behandelt. Aus diesem Patientengut wurde eine Stichprobe genommen, die alle Kinder unter 6 Jahren umfasst, die während eines Zeitraumes von einem Jahr vorgestellt wurden. Die Stichprobe umfasste 106 Kinder, davon 73 Jungen und 33 Mädchen. Das Durchschnittsalter betrug 4 10/12 Jahre. Die Kinder kamen aus allen sozialen Schichten. Die Schichtzugehörigkeit wurde nach Bauer (1972) ermittelt. 33 Kinder wurden der Oberschicht, 36 der Mittelschicht und 37 der Unterschicht zugeordnet.

\subsection{Untersuchungsinstrumente}

\subsubsection{Multiaxiale Diagnosen}

Die Diagnose wurde nach dem multiaxialen Klassifikationsschema für psychische Störungen des Kindes- und Jugendalters nach ICD-10 der WHO gestellt. Zur Diagnosestellung im Rahmen der Achse I (klinisch-psychiatrisches Syndrom) und Achse II (umschriebene Entwicklungsrückstände) wurden neben einer ausführlichen Anamnese auch das Interview „Autism Diagnostic Interview-Revised“ (ADI-R) bzw. das Spielinterview „Autism Diagnostic Observation Schedule“ (ADOS) durch- 
geführt. Zur Beurteilung der Intelligenzleistung (Achse III) wurden das Testverfahren Kaufmann-Assessment Battery for Children (K-ABC) oder der Snijders-Oomen Non-verbale Intelligenzest (SON-R) verwendet. Die somatische Diagnostik (Achse IV) umfasste neben einer intern-pädiatrischen und neurologischen Untersuchung bei den meisten Kindern die Ableitung eines EEGs.

\subsubsection{Child behavior Checklist 11/2-5}

Die Child Behavior Checklist (CBCL) ist ein Fragebogen, der das Urteil von Eltern oder einer Hauptbezugsperson zu Eigenschaften und Verhaltensweisen ihres Kindes erfasst. Verwendet wurde die deutsche Fassung des Fragebogens CBCL 1 1/2-5 (Achenbach u. Rescorla, 2000). Das Ausfüllen des Fragebogens dauert ca. 15 Minuten, und wurde von den Eltern bereitwillig ausgeführt. Der Fragebogen CBCL 1 1/2-5 beinhaltet 100 Fragen (Items) zu Verhaltensauffälligkeiten, somatischen und vegetativen Symptomen und umfasst einen Beurteilungszeitraum von 2 Monaten. Die Fragen werden mit 0 bewertet, wenn die Aussage nicht zutreffend, mit 1, wenn die Aussage etwas/ manchmal zutreffend und mit 2, wenn die Aussage genau/häufig zutreffend ist.

Die Summe der Werte der 100 Items bildet den Gesamtwert (TOT). In der weiteren Auswertung werden verschiedene Aspekte des Verhaltens unterschieden und in acht Problemskalen zusammengefasst: Emotional reaktives Verhalten (SYN I), Angst/Depressivität (SYN II), Körperliche Beschwerden (SYN III), Rückzugsverhalten (SYN IV), Schlafprobleme (SYN V), Aufmerksamkeitsprobleme (SYN VI), Aggressivität (SYN VII), Andere Probleme (Zusatzskala). Zudem werden - im Unterschied zum Vorläufermodell CBCL 2-3 - einige für eine psychiatrische Diagnosestellung besonders relevante Items fünf verschiedenen Diagnoseskalen zugeordnet: Emotionale Störung (DIA I), Angststörung (DIA II), Tiefgreifende Entwicklungsstörung (DIA III), Hyperkinetische Störung (DIA IV) und Sozialverhaltensstörung (DIA V). Diese Diagnoseskalen orientieren sich am Diagnostic and statistical manual of mental disorders (DSM-IV).

Für den jeweiligen Rohwert können anhand einer Tabelle entsprechende Transformationswerte (T-Wert) ermittelt werden, die dann den Vergleich mit einer Normstichprobe ermitteln. Für den Gesamtwert werden T-Werte zwischen 60 und 63 als Grenzbereich, T-Werte über 64 als krankhaft definiert (Achenbach u. Rescorla, 2000). Für die Problem- und Diagnoseskalen werden T-Werte von 65 bis 69 als Grenzbereich, T-Werte von über 70 als krankhaft definiert (Achenbach u. Rescorla, 2000). Nach Sichtung der Daten erwies es sich als sinnvoll, bereits Kinder ab einem T-Wert im Grenzbereich als auffällig einzustufen. Deutsche Normen für die Auswertung liegen nicht vor. Die Ergebnisse einer Überprüfung der psychometrischen Parameter der CBCL 11/2-5 an einer deutschen Stichprobe von insgesamt 246 Kindern im Rahmen einer Inaugural-Dissertation sprechen dafür, dass die „amerikanische Normierung ohne die Gefahr größerer Verfälschungen auch im deutschen Sprachraum angewandt werden kann, bis Normwerte an einer entsprechend repräsentativen Stichprobe vorliegen“ (Elting, 2003, S.2).

(C) Vandenhoeck \& Ruprecht GmbH \& Co. KG, Göttingen 2007 


\subsubsection{Statistische Vorgehensweise}

Zur Auswertung der CBCL-Daten (Mittelwertsvergleiche) wurden folgende statistischen Verfahren verwendet: Der Vergleich von zwei unabhängigen Stichproben bei intervallskalierten Variablen erfolgte mittels t-Test nach Student und anschließender Alpha-Adjustierung des Signifikanzniveaus. Der Vergleich von mehr als zwei unabhängigen Stichproben bei intervallskalierten Variablen erfolgte mittels einfaktorieller Varianzanalyse (Oneway Anova) und dem Post-Hoc-Verfahren Tukey-HSD.

\section{Ergebnisse}

\subsection{Auswertung der klinischen Diagnostik}

Bei 63 Kindern wurde eine klinisch psychiatrische Diagnose (Achse I) gestellt. Die psychiatrischen Hauptdiagnosen wurden vier Diagnosegruppen zugeordnet.

Tabelle 1: Unterteilung der Patienten anhand der klinisch-psychiatrischen Erstdiagnose (Achse I)

\begin{tabular}{llll}
\hline \multicolumn{2}{c}{ Diagnosegruppe } & Klinisch-Psychiatrisches Syndrom (Achse I) & Anzahl \\
\hline 1 & Keine psych.Störung & & 43 \\
\cline { 3 - 4 } & & Kein klinisch-psychiatrisches Syndrom & 21 \\
\hline 2 & Expansive Störung & & 14 \\
& & Einfache Aktivitäts- und Aufmerksamkeitsstörung & 3 \\
& & Hyperkinetische Störung des Sozialverhaltens & 2 \\
& Störung des Sozialverhaltens & 2 \\
\hline & Störung des Sozialverhaltens und der Emotionen & 21 \\
& & 20 \\
& Autismus & Frühkindlicher Autismus & 1 \\
\hline & & Asperger Syndrom & 21 \\
& & Anpassungsstör. mit vorw. Beeinträchtigung v. anderen Gefühlen & 11 \\
& Gefühlsstörung & Anpassungsstörung mit kurzer depressiver Reaktion & 5 \\
& & Emotionale Störung mit Trennungsangst & 1 \\
& & Emotionale Störung mit sozialer Ängstlichkeit & 1 \\
& & Reaktive Bindungsstörung & 1 \\
& & Sonstige Verhaltens/ emotionale Störungen & 2 \\
\hline
\end{tabular}

Eine umschriebene Entwicklungsstörung (Achse II) wurde bei 84 Kindern festgestellt. Bei 22 Kindern wurde keine Diagnose, bei 57 Kindern eine, bei 27 Kindern 2 Diagnosen auf der Achse II vergeben.

(C) Vandenhoeck \& Ruprecht GmbH \& Co. KG, Göttingen 2007 
Die Kinder wurden anhand des Intelligenzniveaus (Achse III) in 3 Gruppen unterteilt. Eine durchschnittliche bis hohe Begabung (IQ > 85) fand sich bei 60 Kindern, eine niedrige Intelligenz (IQ 70-85) bei 27 Kindern und eine leichte Intelligenzminderung $(\mathrm{IQ}<70)$ bei 19 Kindern.

\subsection{Differenzierungsfähigkeit der CBCL 11/2-5 bezüglich der klinisch-psychiatri- schen Diagnose}

\subsubsection{Einfluss Geschlecht, Soziale Schicht und Intelligenz auf den Gesamtwert}

Es zeigte sich kein Zusammenhang zwischen dem Geschlecht und den Mittelwerten des Gesamtwertes ( $t$-Test für unabhängige Stichproben; $F=1,04, \mathrm{df}=104, \mathrm{p}<0,3$ ). Der Mittelwert des Gesamtwertes der Jungen (Mittelwert $M=54$, Standardabweichung $\mathrm{SD}=11)$ war mit dem der Mädchen $(\mathrm{M}=56, \mathrm{SD}=12)$ vergleichbar. Es konnten keine Schichteffekte (einfaktorielle Varianzanalyse; $\mathrm{F}=0,16, \mathrm{df}=105, \mathrm{p}<0,85$ ) festgestellt werden. Bezüglich des Faktors „Intelligenz“ wurden Kinder mit einer durchschnittlichen bis überdurchschnittlichen Begabung mit den Kindern mit einer unterdurchschnittlichen Begabung verglichen. Es konnten keine Intelligenzeffekte ( $t$-Test für unabhängige Stichproben; $F=0,19, \mathrm{df}=104, \mathrm{p}<0,89)$ festgestellt werden.

\subsubsection{Gesamtwert}

Mittels einfaktorieller Varianzanalyse wurde überprüft, inwiefern sich die Mittelwerte für den Gesamtwert für die 4 Diagnosegruppen unterscheiden. Dabei wurde festgestellt, dass Kinder mit einer expansiven Störung $(M=60, S D=9)$ mit der Wahrscheinlichkeit $\mathrm{p}<0,001$ sowie Kinder mit einer autistischen Störung $(\mathrm{M}=61, \mathrm{SD}=10)$ mit $p<0,001$ einen signifikant höheren Gesamtwert als Kinder ohne eine psychiatrische Störung $(M=49, S D=10)$ hatten. Hingegen unterschieden sich Kinder mit einer Gefühlsstörung $(M=55, S D=15)$ von keiner anderen Diagnosegruppe signifikant.

In einem weiteren Schritt wurde überprüft, wie viele Kinder aus den 4 Diagnosegruppen bezüglich des Gesamtwertes den von Achenbach festgelegten Grenzwert von 59 überschritten und somit als auffällig eingeordnet werden müssen.

Jeweils 9 von 21 Kindern (43\%) mit einer expansiven oder autistischen Störung und 6 von 21 Kindern (29\%) mit einer Störung der Gefühle wurden im CBCL als auffällig eingestuft. Die Sensitivität, also die Anzahl der Kinder mit einer psychiatrischen Diagnose, die anhand des Gesamtwertes richtig klassifiziert wurde, liegt somit nur bei knapp 40\%. 5 von 43 Kindern (12\%) ohne eine psychiatrische Diagnose hatten einen auffälligen Gesamtwert. Die Spezifität, dass heißt die Anzahl richtig negativer Klassifikationen, liegt somit bei $88 \%$. Das ergibt für den Gesamtwert eine Fehlklassifizierungsrate von $42 \%$.

Mittels Roc-Kurven wurde ermittelt, ob sich bei einem veränderten Grenzwert die Fehlklassifizierungsrate verringert. Die besten Ergebnisse wurden für einen Schwellenwert von 53 mit einer Spezifität und Sensitivität von jeweils knapp 70\% erreicht. 


\subsubsection{Problemskalen}

Mittels einfaktorieller Varianzanalyse wurden statistisch signifikante Mittelwertsunterschiede zwischen den 4 Diagnosegruppen für die Problemskalen IV-VII festgestellt.

Tabelle 2: Mittelwert und Standardabweichung für SYN I-VII in den 4 Diagnosegruppen

\begin{tabular}{llllll}
\hline & & \multicolumn{4}{c}{ Diagnosegruppen } \\
\cline { 3 - 6 } & & \multicolumn{2}{c}{ 1 } & \multicolumn{1}{c}{3} \\
\cline { 3 - 6 } & & $\begin{array}{c}\text { Keine psych. } \\
\text { Störung }\end{array}$ & $\begin{array}{c}\text { Expansive } \\
\text { Störung }\end{array}$ & Autismus & $\begin{array}{c}\text { Gefühls- } \\
\text { störung }\end{array}$ \\
\cline { 3 - 6 } & & \multicolumn{2}{l}{ Mittelwert (Standardabweichung) } \\
\hline SYN I & Emotional reaktives Verh. & $55(7)$ & $59(10)$ & $58(8)$ & $59(13)$ \\
SYN II & Angst/Depressivität & $54(6)$ & $56(7)$ & $56(8)$ & $57(9)$ \\
SYN III & Körperliche Beschwerden & $55(7)$ & $58(8)$ & $58(9)$ & $56(9)$ \\
SYN IV & Rückzugsverhalten & $59(9)$ & $61(9)$ & $73(8)$ & $62(11)$ \\
SYN V & Schlafprobleme & $52(4)$ & $58(9)$ & $55(8)$ & $55(11)$ \\
SYN VI & Aufmerksamkeitsprobl. & $54(6)$ & $61(8)$ & $60(9)$ & $55(8)$ \\
SYN VII & Aggressivität & $52(4)$ & $61(12)$ & $57(7)$ & $57(11)$ \\
\hline
\end{tabular}

Durch Mehrfachvergleiche mittels Tukey-HSD (Varianzanalysen) wurde ermittelt, zwischen welchen Diagnosegruppen die statistisch signifikante Unterschiede für die Problemskalen IV-VII festzustellen waren: In der Skala „Rückzugsverhalten“ ergab sich jeweils ein höchstsignifikanter Unterschied $(\mathrm{p}<0,001)$ zwischen Kindern mit einer autistischen Störung, sowohl zu den Kindern ohne eine psychiatrische Störung, als auch zu den Kindern mit einer expansiven Störung und den Kindern mit einer Gefühlsstörung. In der Skala „Schlafprobleme“ ergab sich ein signifikanter Unterschied ( $\mathrm{p}=0,032)$ zwischen Kindern mit einer expansiven Störung und den Kindern ohne eine psychiatrische Störung. In der Skala „Aufmerksamkeitsprobleme“ unterschieden sich die Kinder ohne eine psychiatrische Diagnose mit dem niedrigsten Mittelwert sehr signifikant, sowohl von den Kindern mit einer expansiven Störung $(\mathrm{p}=0,001)$, als auch von den autistischen Kindern ( $\mathrm{p}=0,007)$. Die Kinder mit einer Gefühlsstörung hatten einen etwas höheren Mittelwert als die psychiatrisch gesunden Kinder und unterschieden sich somit nur mehr signifikant $(\mathrm{p}=0,046)$ von den hyperkinetischen Kindern. In der Skala „Aggressivität" wurde ein signifikanter Unterschied zwischen Kindern ohne eine psychiatrische Diagnose und Kindern mit einer expansiven Störung $(\mathrm{p}=0,001)$ festgestellt.

Es wurde überprüft, wie viele Kinder aus den 4 Diagnosegruppen den Grenzwert von 64 überschritten und somit als auffällig eingestuft werden.

Kinder ohne eine klinisch psychiatrische Diagnose erreichten am häufigsten einen auffälligen Wert in der Skala „Rückzugsverhalten“. Kinder mit einer expansiven Störung hatten am häufigsten einen auffälligen Wert in der Skala „Aufmerksamkeitsprobleme“, gefolgt von der Skala „Aggressivität“ und der Skala „Emotional re- 
aktives Verhalten“. Kinder mit einer autistischen Störung erzielten am häufigsten einen auffälligen Wert in der Skala „Rückzugsverhalten“, dann in der Skala „Aufmerksamkeitsprobleme“ sowie in der Skala „Emotional reaktives Verhalten“. Kinder mit einer Störung der Gefühle überschritten am häufigsten den Grenzwert in der Skala „Rückzugsverhalten“, gefolgt von der Skala „Emotional reaktives Verhalten“.

Tabelle 3: Anzahl der Kinder mit auffälligem Wert für SYN I-VII in den 4 Diagnosegruppen

\begin{tabular}{|c|c|c|c|c|c|c|c|c|c|}
\hline & & \multicolumn{8}{|c|}{ Diagnosegruppen } \\
\hline & & \multicolumn{2}{|c|}{1} & \multicolumn{2}{|c|}{2} & \multicolumn{2}{|c|}{3} & \multicolumn{2}{|c|}{4} \\
\hline & & \multicolumn{2}{|c|}{$\begin{array}{c}\text { Keine psych. } \\
\text { Störung }\end{array}$} & \multicolumn{2}{|c|}{$\begin{array}{l}\text { Expansive } \\
\text { Störung }\end{array}$} & \multicolumn{2}{|c|}{ Autismus } & \multicolumn{2}{|c|}{$\begin{array}{l}\text { Gefühls- } \\
\text { störung }\end{array}$} \\
\hline & Anzahl & \multicolumn{2}{|c|}{43} & \multicolumn{2}{|c|}{21} & \multicolumn{2}{|c|}{21} & \multicolumn{2}{|c|}{21} \\
\hline \multicolumn{10}{|c|}{ Anzahl T-Wert $>64$} \\
\hline SYN I & Emotional reaktives Verh. & 6 & $14 \%$ & 7 & $33 \%$ & 7 & $33 \%$ & 5 & $24 \%$ \\
\hline SYN II & Angst/Depressivität & 5 & $12 \%$ & 2 & $10 \%$ & 5 & $24 \%$ & 4 & $19 \%$ \\
\hline SYN III & Körperliche Beschwerden & 7 & $16 \%$ & 5 & $24 \%$ & 5 & $24 \%$ & 4 & $19 \%$ \\
\hline SYN IV & Rückzugsverhalten & 10 & $23 \%$ & 6 & $29 \%$ & 18 & $86 \%$ & 10 & $48 \%$ \\
\hline SYN V & Schlafprobleme & 1 & $2 \%$ & 2 & $10 \%$ & 3 & $14 \%$ & 2 & $10 \%$ \\
\hline SYN VI & Aufmerksamkeitsprobleme & 5 & $12 \%$ & 9 & $43 \%$ & 7 & $33 \%$ & 3 & $14 \%$ \\
\hline SYN VII & Aggressivität & 0 & $0 \%$ & 7 & $33 \%$ & 4 & $19 \%$ & 4 & $19 \%$ \\
\hline
\end{tabular}

\subsubsection{Diagnoseskalen}

Mittels einfaktorieller Varianzanalyse wurden statistisch signifikante Mittelwertsunterschiede zwischen den 4 Diagnosegruppen für die Diagnoseskala III-V festgestellt.

Tabelle 4: Mittelwert und Standardabweichung für DIA I-V in den 4 Diagnosegruppen

\begin{tabular}{llllll}
\hline & & \multicolumn{3}{c}{ Diagnosegruppen } \\
\cline { 3 - 6 } & & \multicolumn{2}{c}{ 1 } & \multicolumn{1}{c}{3} & \multicolumn{1}{c}{4} \\
\cline { 3 - 6 } & & $\begin{array}{c}\text { Keine psych. } \\
\text { Störung }\end{array}$ & $\begin{array}{c}\text { Expansive } \\
\text { Störung }\end{array}$ & Autismus & $\begin{array}{c}\text { Gefühls- } \\
\text { störung }\end{array}$ \\
\cline { 3 - 6 } & & Mittelwert (Standardabweichung) & \\
\hline DIA I & Emotionale Störung & $53(7)$ & $56(6)$ & $59(9)$ & $57(11)$ \\
DIA II & Angststörung & $53(5)$ & $56(8)$ & $57(9)$ & $57(11)$ \\
DIA III & Tiefgr. Entw.störung & $62(9)$ & $65(9)$ & $75(7)$ & $64(11)$ \\
DIA IV & Hyperkinetische Störung & $53(5)$ & $61(9)$ & $55(6)$ & $54(6)$ \\
DIA V & Sozialverhaltensstörung & $52(4)$ & $60(10)$ & $58(7)$ & $56(9)$ \\
\hline
\end{tabular}

Durch Mehrfachvergleiche mittels Tukey-HSD (Varianzanalysen) wurde wiederum ermittelt, zwischen welchen Diagnosegruppen die statistisch signifikanten Unterschiede für die Diagnoseskalen III-V festzustellen waren:

(C) Vandenhoeck \& Ruprecht GmbH \& Co. KG, Göttingen 2007 
In der Skala „Tiefgreifende Entwicklungsstörung“ unterschieden sich die autistischen Kinder höchst signifikant von den Kindern ohne eine psychiatrische Störung $(\mathrm{p}<0,001)$, sehr signifikant von den Kindern mit einer expansiven Störung $(\mathrm{p}=0,004)$ und den Kindern mit einer Gefühlsstörung $(\mathrm{p}=0,001)$. In der Skala „Hyperkinetische Störung“ unterschieden sich die Kinder mit einer expansiven Störung höchst signifikant von den Kindern ohne eine psychiatrische Störung $(\mathrm{p}<0,001)$, sehr signifikant von den Kindern mit einer Gefühlsstörung $(\mathrm{p}=0,001)$ und signifikant von den autistischen Kindern $(\mathrm{p}=0,013)$. In der Skala „Sozialverhaltensstörung" unterschieden sich die Kinder mit einer expansiven Störung sehr signifikant (mit $\mathrm{p}=0,002$ ) und die autistischen Kinder (mit $\mathrm{p}=0,019$ ) signifikant von den Kindern ohne eine psychiatrische Diagnose. Es wurde überprüft, wie viele Kinder aus den 4 Diagnosegruppen den Grenzwert von 64 überschritten und somit als auffällig eingestuft werden.

Tabelle 5: Anzahl der Kinder mit auffälligem Wert für DIA I-V in den 4 Diagnosegruppen

\begin{tabular}{|c|c|c|c|c|c|c|c|c|}
\hline & & \multicolumn{7}{|c|}{ Diagnosegruppen } \\
\hline & & 1 & \multicolumn{2}{|c|}{2} & \multicolumn{2}{|c|}{3} & \multicolumn{2}{|c|}{4} \\
\hline & & $\begin{array}{c}\text { Keine psych. } \\
\text { Störung }\end{array}$ & \multicolumn{2}{|c|}{$\begin{array}{l}\text { Expansive } \\
\text { Störung }\end{array}$} & \multicolumn{2}{|c|}{ Autismus } & \multicolumn{2}{|c|}{$\begin{array}{l}\text { Gefühls- } \\
\text { störung }\end{array}$} \\
\hline & Anzahl & 43 & \multicolumn{2}{|c|}{21} & \multicolumn{2}{|c|}{21} & \multicolumn{2}{|c|}{21} \\
\hline \multicolumn{9}{|c|}{ Anzahl T-Wert $>64$} \\
\hline DIA I & Emotionale Störung & $4 \quad 10 \%$ & 0 & $0 \%$ & 6 & $29 \%$ & 5 & $24 \%$ \\
\hline DIA II & Angststörung & $37 \%$ & 2 & $10 \%$ & 3 & $14 \%$ & 5 & $24 \%$ \\
\hline DIA III & Tiefgr. Entw.störung & $1637 \%$ & 13 & $62 \%$ & 21 & $100 \%$ & 9 & $43 \%$ \\
\hline DIA IV & Hyperkinetische Störung & $25 \%$ & 9 & $43 \%$ & 2 & $2 \%$ & 2 & $10 \%$ \\
\hline DIA V & Sozialverhaltensstörung & $12 \%$ & 7 & $33 \%$ & 3 & $14 \%$ & 4 & $19 \%$ \\
\hline
\end{tabular}

Klinisch psychiatrisch gesunden Kindern wurde durch die am DSM-IV orientierten Diagnoseskalen der CBCL $1 \frac{112-5}{2}$ in $37 \%$ die Verdachtsdiagnose einer tiefgreifenden Entwicklungsstörung zugeordnet. Bei Kindern mit der klinischen Diagnose einer expansiven Störung wurde im CBCL in $43 \%$ der Verdacht einer hyperkinetischen Störung sowie in 34\% einer Sozialverhaltensstörung erhoben. Allerdings wurde bei $62 \%$ eine tiefgreifende Entwicklungsstörung vermutet. Bei allen autistischen Kindern wurde in den CBCL-Diagnoseskalen der Verdacht auf eine tiefgreifende Entwicklungsstörung gestellt. Am zweithäufigsten (bei 29\%) wurde eine emotionale Störung vermutet. Kindern mit einer Störung der Gefühle wurde in je $24 \%$ eine emotionale Störung oder Angststörung zugeschrieben. Jedoch am häufigsten (mit 43\%) wurde dieser Diagnosegruppe der Verdacht einer tiefgreifenden Entwicklungsstörung zugeordnet. 


\section{Diskussion}

Eine Stichprobe von 106 Kindern unter 6 Jahren wurde mit 2 Methoden untersucht. Erstens erfolgte eine umfassende klinische kinderpsychiatrische Diagnostik, zweitens füllten die Eltern jeweils den Fragebogen CBCL 1 1/2-5 aus. Durch einen Vergleich der beiden angewandten Methoden sollte festgestellt werden, ob die CBCL 1⁄2-5 zwischen vier klinisch ermittelten Diagnosegruppen unterscheiden kann.

\subsection{Klinische Erkenntnisse}

Es wurden während des Zeitraumes von einem Jahr mehr als doppelt so viele Jungen vorgestellt wie Mädchen. Lavigne et al. (1996) fanden im Vorschulalter ebenfalls signifikant höhere Prävalenzen an Verhaltensproblemen aller Schweregrade bei Jungen (Steinhausen, 2002). Auch in der Studie „Preschool to School“von Richman et al. (1982) wurden häufiger Verhaltensauffälligkeiten bei Jungen als bei Mädchen beobachtet.

Mittels einer multimodalen klinischen Diagnostik wurde bei 63 Kindern eine psychiatrische Erkrankung (Achse I) diagnostiziert. Am häufigsten (bei 20 Kindern) wurde die Diagnose frühkindlicher Autismus gestellt. Frühkindlicher Autismus ist eine seltene Diagnose. Die Häufigkeit wird von der aktuellen Literatur, basierend auf internationalen Studien, mit ca. 1 bis 2 pro 1000 angegeben (Noterdaeme et al., 2002). In die Spezialambulanz werden aber überregional Kinder mit der Verdachtsdiagnose einer tiefgreifenden Entwicklungsstörung zur weiteren Abklärung überwiesen. Somit erklärt sich der überproportionale Anteil autistischer Kinder unter den hier untersuchten Patienten. Am zweithäufigsten (bei 17 Kindern) wurde eine Hyperkinetische Störung diagnostiziert, in knapp 20\% (bei 3 Kindern) bereits in Kombination mit einer Sozialverhaltensstörung. Zählt man die Patienten mit einer Sozialverhaltensstörung oder kombinierten Störung des Sozialverhaltens und der Emotionen hinzu, wurde bei 21 Kindern, also mehr als 30\% der vorgestellten Patienten eine expansive Störung festgestellt. Expansive Störungen sind bereits im Vorschulalter weit verbreitet und überwiegen bei Jungen (Döpfner u. Lehmkuhl, 2002). Am dritthäufigsten (bei 11 Kindern) wurde die Diagnose einer Anpassungsstörung mit vorwiegender Beeinträchtigung von anderen Gefühlen gestellt. Laut ICD-10 umfassen die Symptome verschiedene affektive Qualitäten wie Angst, Depression, Sorgen, Anspannung und Ärger; auch für Kinder mit regressivem Verhalten wird diese Kategorie verwendet. Bei 5 weiteren Patienten wurde die Diagnose einer Anpassungsstörung mit kurzer depressiver Reaktion gestellt. Die Diagnose einer emotionalen Störung wurde nur zweimal gestellt, somit wurde eine Störung von Gefühlen in der Mehrheit der Fälle als Anpassungsstörung verschlüsselt. Zur Epidemiologie von Anpassungsstörungen im Vorschulalter gibt es keine verlässlichen Zahlenangaben. Die Zuordnung einer Anpassungsstörung wird generell kritisch diskutiert. Dennoch liegt die Prävalenz einer Anpassungsstörung in verschiedenen kinderund jugendpsychiatrischen Stichproben bei 7 bis sogar 65\% (Remschmidt, 2000). 
Emotionale Auffälligkeiten werden im Vorschulalter von verschiedenen Autoren als sehr häufig beschrieben (McDonell u. Glod, 2003). Konkrete Angaben, unter welchen Diagnosen diese verschlüsselt werden, fehlen jedoch meistens. Dies spiegelt die Schwierigkeiten wider, Verhaltensstörungen im Vorschulalter zu klassifizieren.

Eine umschriebene Entwicklungsstörung (Achse II) wurde bei 84 Kindern diagnostiziert. Die in der Literatur beschriebene hohe Komorbidität von umschriebenen Entwicklungsstörungen der Sprache und Verhaltensstörungen (Amorosa u. Noterdaeme, 2003) bestätigte sich für das Vorschulalter. Die Hälfte aller Kinder mit einer umschriebenen Entwicklungsstörung des Sprechens und der Sprache litt zugleich unter einer psychiatrischen Störung. Bei Kindern mit einer rezeptiven Sprachstörung war die häufigste Verhaltensstörung eine expansive Störung, gefolgt von einer Gefühlsstörung. Bei Kindern mit einer expressiven Sprachstörung war die Reihenfolge umgekehrt. Verschiedene Autoren berichten von erheblichen emotionalen oder hyperkinetischen Störungen bei Kindern mit einer rezeptiven Sprachstörung. Bei expressiv sprachgestörten Kindern wurden vor allem emotionale Probleme beobachtet (Noterdaeme u. Amorosa, 1998).

\subsection{Aussagekraft des Gesamtwertes}

Der Gesamtwert repräsentiert die Summe aller Items und sollte somit zwischen gesunden Kindern und Kindern mit einer psychiatrischen Diagnose unterscheiden.

Die Anzahl der Kinder mit einer psychiatrischen Diagnose, die anhand des Gesamtwertes richtig klassifiziert wurde (Sensitivität), liegt nur bei 40\%. Aus einem unauffälligen Gesamtwert kann man also wenig Rückschlüsse ziehen. Die Anzahl richtig negativer Klassifikationen (Spezifität) liegt hingegen bei $88 \%$. Ein auffälliger Gesamtwert legt also den Verdacht einer psychiatrischen Störung nahe. Eine Senkung des Schwellenwertes verringert die Fehlklassifizierungsrate.

Betrachtet man die Mittelwerte der Gesamtwerte, erreichten die Kinder mit der Diagnose Autismus die höchsten Werte, gefolgt von den Kindern mit einer expansiven Störung. Beide Gruppen unterschieden sich - im Gegensatz zu Kindern mit einer Gefühlsstörung - jeweils signifikant von Kindern ohne eine psychiatrische Diagnose. Die meisten Kinder, die der Diagnosegruppe Gefühlsstörung zugeordnet waren, hatten eine Anpassungsstörung. Die schlechte Diskriminierung von Kindern mit einer Gefühlsstörung von den anderen Diagnosegruppen stellt auch die klinische Zuordnung einer Anpassungsstörung in Frage. „Aufgrund der fehlenden, klar abgegrenzten operationalen diagnostischen Kriterien ist die Anpassungsstörung keine sehr reliable und valide Diagnose“ (Remschmidt, 2000, S.238-239).

In einer Studie von Noterdaeme und Amorosa (1998) zu Verhaltensauffälligkeiten bei sprachentwicklungsgestörten Kindern ermittelten die Autoren für eine Klassifizierung anhand des im CBCL 4-18 erreichten Gesamtwertes eine Sensitivität von $76 \%$ und eine Spezifität von $88 \%$. Die Fehlklassifizierungsrate lag somit deutlich niedriger als in der hier diskutierten Untersuchung. Angewendet wurde aber die 
CBCL 4-18 und das Durchschnittsalter lag mit 8,5 Jahren deutlich höher. Das Ergebnis ist somit nicht zu einem unmittelbaren Vergleich geeignet. Elting (2003) konzentrierte sich bei der Auswertung der CBCL 1/2-5 auf einen Vergleich der Mittelwerte. Es wurden keine Angaben darüber gemacht, wie viele Patienten aus den einzelnen Diagnosegruppen im Gesamtwert über dem Cut-Off-Wert lagen und somit anhand des Fragebogens tatsächlich als klinisch auffällig eingeschätzt werden. Geeignete Vergleichsdaten bezüglich des CBCL 11/2-5 liegen in der aktuellen Literatur derzeit noch nicht vor.

Haffner et al. (2002) räumten ein, dass der CBCL-Gesamtwert im Einzelfall nicht mit einer Behandlungsbedürftigkeit korreliert, zumal z. B. ein Kind mit wenigen, aber schwer ausgeprägten Symptomen einen niedrigen Gesamtwert erzielen kann. Auch Noterdaeme und Amorosa (1998) empfahlen, nicht allein aus der Beurteilung des Gesamtwertes Rückschlüsse zu ziehen. Neben dem Gesamtwert sollten bestimmte Symptomkonstellationen in der CBCL als Anlass zu einer exakten individuellen psychiatrischen Diagnostik herangezogen werden.

\subsection{Aussagekraft der Problem- und Diagnoseskalen}

Die für die jeweiligen Diagnosegruppen typischen Verhaltensprobleme werden von den Problemskalen zutreffend abgebildet. Die Sensitivität der Problemskalen ist jedoch bis auf die Skala „Rückzugsverhalten“ niedrig. Die Aussagekraft ist bei der Auswertung eines im Problemskalenbereich unauffälligen Fragebogens somit nur gering.

Erstmalig wurden im CBCL 1 1/2-5 Diagnoseskalen eingeführt, die einen Vergleich mit DSM-Diagnosen ermöglichen. Achenbach (2000) verweist jedoch darauf, dass ein auffälliger Wert in den Diagnoseskalen nicht als Äquivalent zu einer DSM-Diagnose aufzufassen ist. Die Mittelwertsvergleiche zeigen, dass die Diagnoseskalen die Kernsymptomatik der jeweiligen Diagnosegruppe erfassen. Die Sensitivität der Diagnoseskalen ist jedoch bis auf die Skala „Tiefgreifende Entwicklungsstörung" niedrig. Alle 21 autistischen Patienten hatten einen auffälligen Wert in der Skala „Tiefgreifende Entwicklungsstörung" und wurden somit anhand der CBCL zutreffend diagnostisch eingeordnet. Die Mittelwerte der autistischen Patienten unterschieden sich in dieser Skala sehr bis höchst signifikant von allen anderen Störungsbildern. Die CBCL kann also bei der Abklärung einer autistischen Störung durchaus zutreffende Hinweise geben, auch wenn es sicher einer nachfolgend differenzierten klinischen Abklärung bedarf. Die Skala „Tiefgreifende Entwicklungsstörung" erzielte jedoch mit 57\% eine schlechte Spezifität. So hatten auch die Kinder ohne eine psychiatrische Störung die höchsten Mittelwerte in dieser Skala III. 41 von 43 Kindern ohne eine psychiatrische Diagnose leiden jedoch an einer umschriebenen Entwicklungsstörung. Die Skala „Tiefgreifende Entwicklungsstörung" differenziert demnach nicht ausreichend zwischen unterschiedlichen Störungsbildern. Bei einem auffallenden Wert in dieser Skala ist demnach eine weitere Abklärung dringend indiziert. 
Kuschel et al. (2006) ermittelten die Prävalenzrate hyperkinetischer Symptome und Störungen in einer Stichprobe von 280 Kindern sowohl mit dem Fragebogen FBB-HKS als auch der CBCL 1 1/2-5. Dabei zeigten die Ergebnisse der CBCL ebenfalls eine nur geringe Sensitivität (bei guter Spezifität) für die Problemskala „Aufmerksamkeitsstörungen“ und noch geringer für die Diagnoseskala „Hyperkinetische Störung“. Nur jedes vierte Kind, das mittels eines für das Vorschulalter modifizierten Fragebogens des FBB-HKS (ermittelte Prävalenz 9,9\%) die Diagnose einer hyperkinetischen Störung zugeschrieben bekam erhielt diese auch in der CBCL 11/2-5 (ermittelte Prävalenz 2,7\%).

Problem- und Diagnoseskalen eigneten sich nicht hinreichend zur Diagnosestellung, gaben aber Hinweise auf komorbide Störungen. So erzielten Kinder mit einer expansiven Störung neben den Skalen „Aufmerksamkeitsprobleme“ und „Aggressivität“ die höchsten Mittelwerte in der Skala „Emotional reaktives Verhalten“. Es ist auch in der Literatur beschrieben, dass bei Kindern mit expansiven Störungen häufig zusätzlich emotionale Probleme auftreten (Lehmkuhl u. Döpfner, 2003). Kinder mit einer autistischen Störung erzielten neben der Skala „Rückzugsverhalten“ die höchsten Mittelwerte in den Skalen „Emotional reaktives Verhalten“ und „Aufmerksamkeitsprobleme“. Wie aus der Literatur bekannt, sind dies typische komorbide Probleme autistischer Kinder (Noterdaeme et al., 2002).

Zusammenfassend fällt bei der Anwendung der CBCL 11/2-5 in einer Spezialambulanz für Verhaltens- und Entwicklungsstörungen die geringe Sensitivität für den Gesamtwert sowie für die Problem- und Diagnoseskalen (mit Ausnahme der Skala „Tiefgreifende Entwicklungsstörung) auf. Die Skala „Tiefgreifende Entwicklungsstörung “ zeigte eine sehr gute Sensitivität, konnte aber nicht ausreichend zwischen einer umschriebenen und tiefgreifenden Entwicklungsstörung differenzieren. Es wäre in weiteren Studien zu klären ob sich diese beiden Ergebnisse bestätigen. Eventuell könnte eine Senkung (bzw. Anhebung bei der Skala „Rückzugsverhalten“) der Schwellenwerte zu einer niedrigeren Fehlklassifizierungsrate führen.

\section{Literatur}

Achenbach,T. M., Rescorla, L.A. (2000). Manual for the ASEBA Preschool Forms \& Profiles. Burlington: University of Vermont.

American Psychiatric Association (2000). Diagnostic and statistical manual of mental disorders. Washington D.C.

Amorosa, H., Noterdaeme, M. (1999). Früherkennung von Entwicklungsstörungen im Kindesalter. Forum der Kinder- und Jugendpsychiatrie und Psychotherapie, 1, 59-65.

Amorosa, H. (2003). Beschreibung der Störung. In H. Amorosa, M. Noterdaeme (Hrsg.), Rezeptive Sprachstörungen (S.10-14). Göttingen: Hogrefe.

Bauer, A. (1972). Ein Verfahren zur Messung des für Bildungsverfahren relevanten sozialen Status. Frankfurt: Deutsches Institut für Internationale Pädagogische Förderung. 
Campbell, S. (1995). Behavior Problems in Preschool Children: A Review of Recent Research. J. Child Psychol. Psychiat, 36, 113-149.

Döpfner, M., Lehmkuhl, G., Heubrock, D., Petermann, F. (2000). Diagnostik psychischer Störungen im Kindes- und Jugendalter. Göttingen: Hogrefe.

Elting, P. (2003). Überprüfung der psychometrischen Parameter von CBCL 11/2-5 und C-TRF an einer deutschen Stichprobe. Dissertation. Johann Wolfgang von Goethe Universität Frankfurt am Main.

Esser, G., Wyschkon, A. (2002). Umschriebene Entwicklungsstörungen. In F. Petermann (Hrsg.), Lehrbuch der klinischen Kinderpsychologie und -psychotherapie (S. 410). Göttingen: Hogrefe.

Goodman, R., Scott, S., Rothenberger, A. (2000). Kinderpsychiatrie kompakt. Darmstadt: Steinkopff.

Haffner,J., Esther, C., Münch, H., Parzer, P., Raue, B., Stehen, R., Klett, M., Resch, F. (2002). Verhaltensauffälligkeiten im Einschulungsalter aus elterlicher Perspektive- Ergebnisse zu Prävalenz und Risikofaktoren in einer epidemiologischen Studie. Praxis der Kinderpsychologie und Kinderpsychiatrie, 51, 675-696.

Herpertz-Dahlmann, B., Resch, F., Schulte-Markwort, M., Warnke, A. (2003). Entwicklungspsychiatrie. In B. Herpertz-Dahlmann et al. (Hrsg.), Entwicklungspsychiatrie (S. 315-357). Stuttgart: Schattauer.

Hofstra, M., Van der Ende, J., Verhulst, F. (2000). Continuity and change of psychopathology from Childhood into Adulthood: A 14-Year Follow-up Study. J.Am.Acad. Child Adolesc. Psychiatry, 39, 7.

Kuschel, A., Lübke, A., Köppe, E., Miller,Y., Hahlweg, K., Sanders, M.R. (2004). Häufigkeit psychischer Auffälligkeiten und Begleitsymptome bei drei- bis sechsjährigen Kindern: Ergebnisse der Braunschweiger Kindergartenstudie. Zeitschrift für Kinder- und Jugendpsychiatrie und Psychotherapie, 32 97-106.

Kuschel, A., Ständer, D., Bertram, H., Heinrichs, N., Naumann, S., Hahlweg, K. (2006). Prävalenz hyperkinetischer Symptome und Störungen im Vorschulalter- ein Vergleich zweier Diagnose-instrumente. Zeitschrift für Kinder- und Jugendpsychiatrie und Psychotherapie, 34, 275-286.

McDonnell, M., Glod, C. (2003). Prevalence of Psychopathology in Preschool-Age Children. J. of Child and Adolescent Psychiatric Nursing, 16, 141-152.

Noterdaeme, M., Amorosa, H. (2002). Früherkennung autistischer Störungen. Monatsschrift Kinderheilkunde, 150, 149-156.

Noterdaeme, M.; Amorosa, H. (1998). Verhaltensauffälligkeiten bei sprachentwicklungsgestörten Kindern, Child-behavior-Checklist als Screening-Instrument. Monatsschrift Kinderheilkunde, 146, 931-937.

Noterdaeme, M., Minow, F., Amorosa, H. (1999). Anwendbarkeit der Child Behavior Checklist bei entwicklungsgestörten Kindern. Zeitschrift für Kinder- und Jugendpsychiatrie, 27, 183-188.

Poustka, F. (1993). Verhaltensauffälligkeiten: Klassifikation, Epidemiologie, Pathogenese und Prognose. In M. Döpfner, M. Schmidt (Hrsg.), Kinderpsychiatrie Vorschulalter (S. 1-16). München: Quintessenz.

Resch, F. (1996). Probleme des Kleinkind- und Vorschulalters. In F. Resch, Entwicklungspsychopathologie des Kindes- und Jugendalters (S. 224-228). Weinheim: Psychologie Verlags Union. 
Remschmidt, H. (2000). Kinder- und Jugendpsychiatrie. Stuttgart: Georg Thieme Verlag.

Remschmidt, H., Schmidt, M. (2001). Multiaxiales Klassifikationsschema für psychische Störungen des Kindes- und Jugendalters nach ICD-10 der WHO. Bern: Huber.

Richman, N., Stevenson, J., Graham, P.J. (1982). Preschool to School: A Behavioural Study. London: Academic Press.

Steinhausen H.Ch. (2002). Psychische Störungen bei Kindern und Jugendlichen. München: Urban \& Fischer.

Suchodoletz, W. von (2003). Umschriebene Sprachentwicklungsstörungen. Monatsschrift Kinderheilkunde, 151, 31-37.

Wolff Metternich,T., Döpfner, M. (2000). Oppositionelle Verhaltensstörungen im Vorschulalter. Kindheit und Entwicklung, 9, 30-39.

Korrespondenzadresse: PD. Dr. Michele Noterdaeme, Heckscher-Klinik für Kinder- und Jugendpsychiatrie und Psychotherapie, Deisenhofener Strasse 28, 81539 München. 